\title{
Selective 6-endo-Cyclization of the Acyl Radicals onto the Nitrogen of Imine and Oxazoline C-N Bonds
}

\author{
Mami Tojino, Noboru Otsuka, Takahide Fukuyama, Hiroshi Matsubara, and Ilhyong Ryu* \\ Department of Chemistry, Graduate School of Science, Osaka Prefecture University, Sakai, Osaka \\ 599-8531, Japan
}

General information. ${ }^{1} \mathrm{H}$ NMR spectra were recorded with a JEOL JMN-500 (500 $\left.\mathrm{MHz}\right)$ spectrometer in $\mathrm{CDCl}_{3}$. Chemical shifts are reported in parts per million $(\delta)$ downfield from internal TMS at $0.00 .{ }^{13} \mathrm{C}$ NMR spectra were recorded with a JEOL JMN ECP-500 (125 MHz) spectrometer or JEOL AL-400 (100 MHz) spectrometer and referenced to the solvent peak at $77.00 \mathrm{ppm}$. Infrared spectra were obtained on a JASCO FT/IR 4100 spectrometer; absorption data are reported in reciprocal centimeters. Conventional and high-resolution mass spectra were obtained on a JEOL MS700 spectrometer. Products were purified by flash chromatography on silica gel (nacalai tesque int. Silica Gel 60, 230-400 mesh) and if necessary, were further purified by recycling preparative HPLC (JAI LC908) equipped with a GPC column using $\mathrm{CHCl}_{3}$ as eluent.

\section{1-Ethylpropyl (4-phenylselenylbutylidene)amine (1a).}

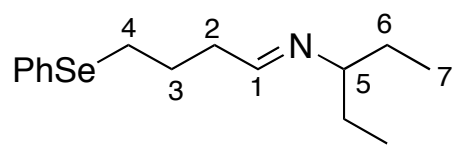

4-(Phenylseleno)butanal $(8.9 \mathrm{mmol}, 2.01 \mathrm{~g})$ was added to 3 -aminopentane $(11.1 \mathrm{mmol}, 920 \mathrm{mg})$ at $0{ }^{\circ} \mathrm{C}$. The mixture was allowed to worm to room temperature and stirred for $30 \mathrm{~min}$. The mixture dried over $\mathrm{Na}_{2} \mathrm{SO}_{4}$. After filtration, the solvent was evaporated. The residue was distilled under reduced pressure to give $1.56 \mathrm{~g}(60 \%)$ of $\mathbf{1 a}\left(122-123{ }^{\circ} \mathrm{C} / 0.3 \mathrm{mmHg}\right) .{ }^{1} \mathrm{H} \mathrm{NMR}\left(500 \mathrm{MHz}, \mathrm{CDCl}_{3}\right) \delta 0.75(\mathrm{t}, J=7.3 \mathrm{~Hz}$, $6 \mathrm{H}, H-7$ ), $1.41-1.57$ (m, 4H, $H-6$ ), 1.93 (qui, $J=7.3 \mathrm{~Hz}, 2 \mathrm{H}, H-3$ ), 2.34-2.38 (m, $2 \mathrm{H}, H-4$ ), 2.59 (sept, $J$ $=4.1 \mathrm{~Hz}, 1 \mathrm{H}, H-5), 2.93(\mathrm{t}, J=7.3 \mathrm{~Hz}, 2 \mathrm{H}, H-2), 7.19-7.25(\mathrm{~m}, 3 \mathrm{H}, H-1,-P h), 7.45-7.48(\mathrm{~m}, 2 \mathrm{H}, H-$ $P h), 7.53(\mathrm{t}, J=4.8 \mathrm{~Hz}, 1 \mathrm{H}, H-P h) ;{ }^{13} \mathrm{C}$ NMR $\left(125 \mathrm{MHz}, \mathrm{CDCl}_{3}\right) \delta 11.01(C-7), 27.14,27.23,28.69$, 35.80, $76.91(C-5), 126.84(C-P h), 129.11(C-P h), 130.27(C-P h), 132.56(C-P h), 162.07(C-1)$; IR (neat) 3057, 2961, 2930, 2872, 2833, 1666, 1579, 1478, 1456, 1438; MS (EI) $\mathrm{m} / z$ (rel intensity) 297 $\left(\mathrm{M}^{+}, 12\right), 157$ (14), 140 (10), 113 (95), 84 (100); HRMS (EI) $\mathrm{m} / z$ calcd for $\mathrm{C}_{15} \mathrm{H}_{23} \mathrm{NSe}\left(\mathrm{M}^{+}\right)$297.0996, found 297.0992 .

\section{1-Ethylpropyl-((1-prop-2-ynylcyclohexyl)methylene) amine (1d).}

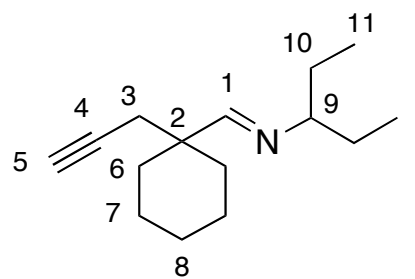

1-Prop-2-ynyl-cyclohexanecarbaldehyde $(9.1 \mathrm{mmol}, 1.37 \mathrm{~g})$ was added to 3 -aminopentane $(9.1 \mathrm{mmol}$, $1.1 \mathrm{~mL}$ ) at $0{ }^{\circ} \mathrm{C}$. The mixture was allowed to worm to room temperature and stirred for $2 \mathrm{~h}$. The mixture dried over $\mathrm{Na}_{2} \mathrm{SO}_{4}$. After filtration, the solvent was evaporated. The residue was distilled 
under reduced pressure to give $1.00 \mathrm{~g}(50 \%)$ of $1 \mathrm{e}\left(62-63{ }^{\circ} \mathrm{C} / 0.3 \mathrm{mmHg}\right) .{ }^{1} \mathrm{H}$ NMR $(500 \mathrm{MHz}$, $\left.\mathrm{CDCl}_{3}\right) \delta 0.76(\mathrm{t}, J=7.3 \mathrm{~Hz}, 6 \mathrm{H}, H-11), 1.22-1.30(\mathrm{~m}, 1 \mathrm{H}), 1.40-1.56(\mathrm{~m}, 11 \mathrm{H}), 1.80-1.86(\mathrm{~m}, 2 \mathrm{H})$, $1.92(\mathrm{t}, J=2.8 \mathrm{~Hz}, 1 \mathrm{H}, H-5), 2.25$ (d, $J=2.8 \mathrm{~Hz}, 2 \mathrm{H}, H-3), 2.62(\mathrm{sep}, J=4.4 \mathrm{~Hz}, 1 \mathrm{H}, H-9), 7.46(\mathrm{~s}, 1 \mathrm{H}$, $H-1) ;{ }^{13} \mathrm{C}$ NMR $\left(125 \mathrm{MHz}, \mathrm{CDCl}_{3}\right) \delta 11.22(C-11), 22.40,25.91,28.86,29.55,33.57,41.69,70.31(C-$ 5), 75.42 (C-2), 81.34 (C-4), 168.22 (C-1); IR (neat) 3312, 2118, 1664; MS (EI) $m / z$ (rel intensity) 219 $\left(\mathrm{M}^{+}, 4\right), 150$ (100); HRMS (EI) $\mathrm{m} / z$ calcd for $\mathrm{C}_{15} \mathrm{H}_{25} \mathrm{~N}\left(\mathrm{M}^{+}\right)$219.1987, found 219.1996.

\section{1-Ethylpropyl (4-iodo-2,2-dimethyl-3-butenylidene) amine (1j).}

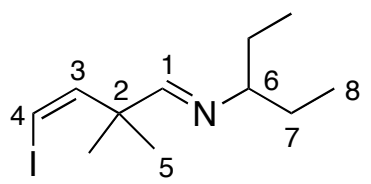

The mixture of (Z)-4-iodo-2,2-dimethyl-3-butenal (4.5 mmol, $1.0 \mathrm{~g}$ ), 3-aminopentane (5.1 mmol, 0.6 $\mathrm{mL})$ and $\mathrm{K}_{2} \mathrm{CO}_{3}(10 \mathrm{~g})$ in $\mathrm{Et}_{2} \mathrm{O}(50 \mathrm{~mL})$ was stirred at room temperature for $19.5 \mathrm{~h}$. After filtration, the solvent was evaporated. The residue was distilled under reduced pressure to give $0.4 \mathrm{~g}(30 \%)$ of $\mathbf{1 j}$ $\left(82{ }^{\circ} \mathrm{C} / 2 \mathrm{mmHg}\right) .{ }^{1} \mathrm{H} \mathrm{NMR}\left(400 \mathrm{MHz}, \mathrm{CDCl}_{3}\right) \delta 0.80(\mathrm{t}, J=7.4 \mathrm{~Hz}, 6 \mathrm{H}, H-8), 1.37$ (s, $\left.6 \mathrm{H}, H-5\right), 1.49-$ $1.60(\mathrm{~m}, 4 \mathrm{H}, H-7), 2.72(\mathrm{sex}, J=4.9 \mathrm{~Hz}, 1 \mathrm{H}, H-6), 6.31(\mathrm{~d}, J=8.3 \mathrm{~Hz}, 1 \mathrm{H}, H-3), 6.82(\mathrm{~d}, J=8.3 \mathrm{~Hz}$, $1 \mathrm{H}, H-4), 7.72(\mathrm{~s}, 1 \mathrm{H}, H-1) ;{ }^{13} \mathrm{C} \mathrm{NMR}\left(100 \mathrm{MHz}, \mathrm{CDCl}_{3}\right) \delta 11.13(C-8), 25.71(C-5), 28.56(C-7)$, $42.41(C-2), 74.39(C-6), 77.86(C-4), 146.18$ (C-3), 167.43 (C-1); IR (neat) 1662, 1602, 1460; MS (EI) $m / z$ (rel intensity) $293\left(\mathrm{M}^{+}, 7\right), 264$ (6), 226 (16), 195(21), 166 (35), 153 (23), 98 (43), 84 (49), 71 (100); HRMS (EI) $m / z$ calcd for $\mathrm{C}_{11} \mathrm{H}_{20} \mathrm{NI}\left(\mathrm{M}^{+}\right)$293.0640, found 293.0633.

Imines 1b, 1c and 1i were prepared by a similar procedure from the corresponding amines and aldehydes. Azaenyne 1e, 1f, $1 \mathrm{~g}$ and $\mathbf{1 h}$ were prepared from a corresponding amines and hex-5-yn-2one by azeotropic distillation using Dean-Stark tube (solvent $\mathrm{C}_{6} \mathrm{H}_{6}$ ).

(R)-4-Phenyl-2-(3-phenylselenylpropyl)-4,5-dihydrooxazole (1m).

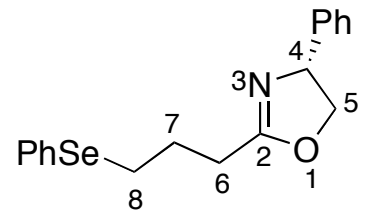

${ }^{1} \mathrm{H}$ NMR $\left(500 \mathrm{MHz}, \mathrm{CDCl}_{3}\right.$ ) $\delta 2.10$ (qui, $\left.J=7.3 \mathrm{~Hz}, 2 \mathrm{H}, H-7\right), 2.51-2.54(\mathrm{~m}, 2 \mathrm{H}, H-8), 3.02(\mathrm{t}, J=7.3$ $\mathrm{Hz}, 2 \mathrm{H}, H-6), 4.05$ (t, $J=8.3 \mathrm{~Hz}, 1 \mathrm{H}, H-5), 4.58$ (t, $J=9.2 \mathrm{~Hz}, 1 \mathrm{H}, H-5), 5.15$ (t, $J=9.2 \mathrm{~Hz}, 1 \mathrm{H}, H-4)$, $7.20(\mathrm{~m}, 6 \mathrm{H}, P h), 7.31(\mathrm{~m}, 2 \mathrm{H}, P h), 7.49(\mathrm{~m}, 2 \mathrm{H}, P h) ;{ }^{13} \mathrm{C} \mathrm{NMR}\left(100 \mathrm{MHz}, \mathrm{CDCl}_{3}\right) \delta 26.40(C-6$ or 7 or 8), 27.01 (C-6 or 7 or 8$), 27.79$ (C-6 or 7 or 8$), 69.53(C-4$ or 5), 74.43 (C-4 or 5), 126.46, 126.79, $127.45,128.64,129.00,132.55,142.32,167.82$ (C-2); IR (neat) 1665, 1578; MS (EI) $\mathrm{m} / z$ (rel intensity) $345\left(\mathrm{M}^{+}, 12\right), 188$ (12), 161 (100), 120 (11), 91 (11); HRMS (EI) m/z calcd for $\mathrm{C}_{18} \mathrm{H}_{19} \mathrm{NOSe}\left(\mathrm{M}^{+}\right)$ 345.0632 , found

345.0623 .

Typical procedure for radical carbonylation. A magnetic stirring bar, AIBN (2,2'azobisisobutyronitrile, $17.3 \mathrm{mg}, 0.1 \mathrm{mmol})$, benzene $(17 \mathrm{~mL})$, imine 1a $(143.6 \mathrm{mg}, 0.48 \mathrm{mmol})$ and $\mathrm{Bu}_{3} \mathrm{SnH}(186.1 \mathrm{mg}, 0.64 \mathrm{mmol})$ were placed in a $50 \mathrm{~mL}$ stainless steel autoclave. The autoclave was closed, purged three times with carbon monoxide, pressurized with 80 atm of $\mathrm{CO}$ and then heated $90{ }^{\circ} \mathrm{C}$ 
for $1 \mathrm{~h}$. Excess $\mathrm{CO}$ was discharged at room temperature. The solvent was removed under reduced pressure. The residue was purified by flash chromatography on silica gel (gradient from hexane to hexane $/ \mathrm{EtOAc}=1 / 1)$ to give $\mathbf{2 a}(66.3 \mathrm{mg}, 81 \%)$.

\section{1-(1-ethylpropyl)piperidin-2-one (2a).}

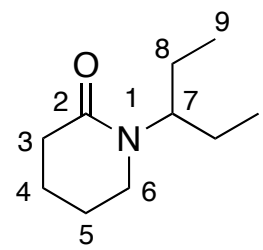

$\left(\mathrm{R}_{\mathrm{f}}=0.28\right.$, hexane/EtOAc $\left.=1 / 1\right) .{ }^{1} \mathrm{H}$ NMR $\left(500 \mathrm{MHz}, \mathrm{CDCl}_{3}\right) \delta 0.83(\mathrm{t}, J=7.3 \mathrm{~Hz}, 6 \mathrm{H}, H-9), 1.36-$ $1.51(\mathrm{~m}, 4 \mathrm{H}, H-8), 1.73-1.79(\mathrm{~m}, 4 \mathrm{H}, H-4,5), 2.43(\mathrm{t}, J=6.9 \mathrm{~Hz}, 2 \mathrm{H}, H-3), 3.03(\mathrm{t}, J=6.0 \mathrm{~Hz}, 2 \mathrm{H}, H-$ 6), 4.53 (sept, $J=5.0 \mathrm{~Hz}, 1 \mathrm{H}, H-7) ;{ }^{13} \mathrm{C} \mathrm{NMR}\left(125 \mathrm{MHz}, \mathrm{CDCl}_{3}\right.$ ) $\delta 10.79$ (C-9), 21.05, 23.22, 24.68, 32.44, $40.39(C-6), 54.92(C-7), 170.42$ (C-2); IR (neat) 1636; MS (EI) $\mathrm{m} / z$ (rel intensity) $169\left(\mathrm{M}^{+}, 18\right)$, 140 (100), 112 (13); HRMS (EI) $m / z$ calcd for $\mathrm{C}_{10} \mathrm{H}_{19} \mathrm{NO}\left(\mathrm{M}^{+}\right)$169.1467, found 169.1465.

Typical procedure for the stannylcarbonylation of alkynyl imine and subsequent protodestannylation. A magnetic stirring bar, AIBN (2,2'-azobisisobutyronitrile, $16.8 \mathrm{mg}, 0.1 \mathrm{mmol}$ ), benzene $(17 \mathrm{~mL})$, alkynyl imine $1 \mathbf{b}(78.6 \mathrm{mg}, 0.52 \mathrm{mmol})$ and $\mathrm{Bu}_{3} \mathrm{SnH}(212.6 \mathrm{mg}, 0.73 \mathrm{mmol})$ were placed in a $50 \mathrm{~mL}$ stainless steel autoclave. The autoclave was closed, purged three times with carbon monoxide, pressurized with 85 atm. of $\mathrm{CO}$ and then heated $90{ }^{\circ} \mathrm{C}$ for $8 \mathrm{~h}$. Excess $\mathrm{CO}$ was discharged at room temperature. The solvent was removed under reduced pressure. The residue was dissolved into $\mathrm{MeOH}(3.5 \mathrm{~mL})$, and TMSCl $(0.42 \mathrm{~mL})$ was added. The solution was stirring at room temperature for $5 \mathrm{~min}$, and evaporated under reduced pressure. The residue was purified by flash chromatography on silica gel (gradient from hexane to hexane/EtOAc $=2 / 1)$ to give $\mathbf{2 b}(67.2 \mathrm{mg}, 71 \%)$.

\section{1-(1-Ethylpropyl)-3-methylene-piperidin-2-one (2b).}

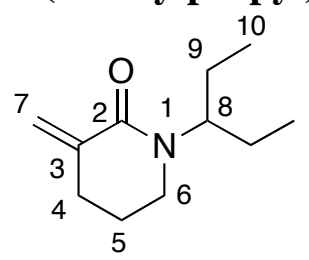

$\left(\mathrm{R}_{\mathrm{f}}=0.35\right.$, hexane/EtOAc $\left.=2 / 1\right) .{ }^{1} \mathrm{H}$ NMR $\left(500 \mathrm{MHz}, \mathrm{CDCl}_{3}\right) \delta 0.86(\mathrm{td}, J=7.3,1.3 \mathrm{~Hz}, 6 \mathrm{H}, H-10)$, 1.40- 1.56 (m, 4H, H-9), 1.86 (qui, $J=6.4,5.7 \mathrm{~Hz}, 2 \mathrm{H}, H-5), 2.58(\mathrm{td}, J=6.4,1.4 \mathrm{~Hz}, 2 \mathrm{H}, H-4), 3.14(\mathrm{t}$, $J=5.7 \mathrm{~Hz}, 2 \mathrm{H}, H-6), 4.60(\mathrm{~m}, 1 \mathrm{H}, H-8), 5.29(\mathrm{t}, J=1.8 \mathrm{~Hz}, 1 \mathrm{H}, H-7), 6.24(\mathrm{t}, J=1.8 \mathrm{~Hz}, 1 \mathrm{H}, H-7)$; ${ }^{13} \mathrm{C}$ NMR $\left(125 \mathrm{MHz}, \mathrm{CDCl}_{3}\right) \delta 10.92(C-10), 23.34(C-5), 24.92(C-9), 29.97(C-4), 41.18(C-6), 55.86$ $(C$-8), $121.78(C-7), 137.95(C$-3), 164.86 (C-2); IR (neat) 1653, 1614; MS (EI) $\mathrm{m} / z$ (rel intensity) 181 (M+1 19), 153 (40), 152 (100), 149 (23), 136 (25), 107 (31), 106 (23), 105 (19), 89 (34), 78 (24), 77 (70), 69 (26), 57 (31), 55 (31), 51 (22); HRMS (EI) $m / z$ calcd for $\mathrm{C}_{11} \mathrm{H}_{19} \mathrm{NO}\left(\mathrm{M}^{+}\right)$181.1467, found 181.1463. 


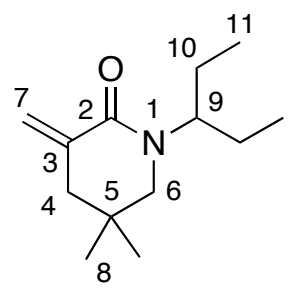

$\left(\mathrm{R}_{\mathrm{f}}=0.14\right.$, hexane/EtOAc $\left.=5 / 1\right) .{ }^{1} \mathrm{H} \mathrm{NMR}\left(500 \mathrm{MHz}, \mathrm{CDCl}_{3}\right) \delta 0.86(\mathrm{t}, J=7.3 \mathrm{~Hz}, 6 \mathrm{H}, H-11), 1.01(\mathrm{~s}$, 6H, H-8) 1.36- 1.53 (m, 4H, H-10), 2.35 (s, 2H, H-4), 2.85 (s, 2H, H-6), 4.57 (broad, 1H, H-9), 5.27 (qua-like, $J=1.8 \mathrm{~Hz}, 1 \mathrm{H}, H-7), 6.26$ (t-like, $J=1.8 \mathrm{~Hz}, 1 \mathrm{H}, H-7) ;{ }^{13} \mathrm{C} \mathrm{NMR}\left(125 \mathrm{MHz}, \mathrm{CDCl}_{3}\right) \delta 11.03$ (C-11), 24.80, 26.26, 30.14, 43.32, 52.96, 55.89, 122.97 (C-7), 136.77 (C-3), 164.49 (C-2); IR (neat) 1659, 1615; MS (EI) m/z (rel intensity) $209\left(\mathrm{M}^{+}, 12\right), 180$ (100), 166 (6); HRMS (EI) $\mathrm{m} / z$ calcd for $\mathrm{C}_{13} \mathrm{H}_{23} \mathrm{NO}\left(\mathrm{M}^{+}\right)$209.1780, found 209.1772.

\section{2-(1-Ethylpropyl)-4-methylene-2-aza-spiro[5.5] undecan-3-one (2d).}

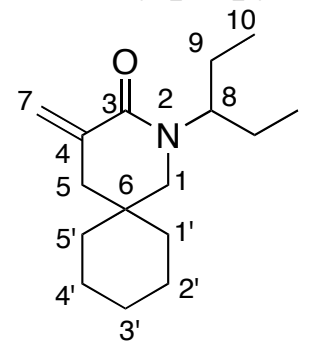

$\left(\mathrm{R}_{\mathrm{f}}=0.23\right.$, hexane/EtOAc $\left.=5 / 1\right) .{ }^{1} \mathrm{H}$ NMR $\left(500 \mathrm{MHz}, \mathrm{CDCl}_{3}\right) \delta 0.85(\mathrm{t}, J=7.3 \mathrm{~Hz}, 6 \mathrm{H}, H-10), 1.23-$ 1.53 (m, 14H, H-1', 2', 3', 4', 5', 9), 2.41 (s, 2H, H-5), 2.89 (s, 2H, H-1), 4.56 (broad, 1H, H-8), 5.27 (t-like, $J=1.8 \mathrm{~Hz}, 1 \mathrm{H}, H-7), 6.23(\mathrm{t}-$ like, $J=1.8 \mathrm{~Hz}, 1 \mathrm{H}, H-7) ;{ }^{13} \mathrm{C} \mathrm{NMR}\left(125 \mathrm{MHz}, \mathrm{CDCl}_{3}\right) \delta 10.99$ (C-10), 21.52 (C-2', 4'), 24.89 (C-9), 26.32, 32.42, 34.48, 40.20, 51.25, 55.83 (C-8), 122.86 (C-7), 136.31 (C-4), 164.72 (C-3); IR (neat) 1659, 1616; MS (EI) $\mathrm{m} / z$ (rel intensity) $249\left(\mathrm{M}^{+}, 9\right), 221$ (16), 220 (100), 206 (6), 81 (4), 79 (6), 77 (4), 67 (6), 55 (6); HRMS (EI) m/z calcd for $\mathrm{C}_{16} \mathrm{H}_{27} \mathrm{NO}\left(\mathrm{M}^{+}\right)$ 249.2093, found 249.2085.

\section{1-(1-Ethylpropyl)-6-methyl-3-methylene-piperidin-2-one (2e).}

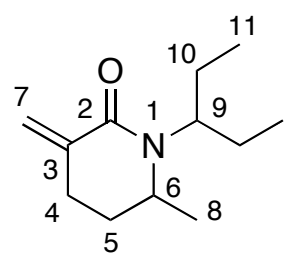

$\left(\mathrm{R}_{\mathrm{f}}=0.29\right.$, hexane/EtOAc $\left.=2 / 1\right) .{ }^{1} \mathrm{H} \mathrm{NMR}\left(500 \mathrm{MHz}, \mathrm{CDCl}_{3}\right) \delta 0.86(\mathrm{t}, J=7.3 \mathrm{~Hz}, 3 \mathrm{H}, H-11), 0.92(\mathrm{t}$, $J=7.3 \mathrm{~Hz}, 3 \mathrm{H}, H-11), 1.25(\mathrm{~d}, J=6.9 \mathrm{~Hz}, 3 \mathrm{H}, H-8), 1.51-1.74(\mathrm{~m}, 5 \mathrm{H}, H-5,10), 1.88-1.95(\mathrm{~m}, 1 \mathrm{H}, H-$ 5), 2.54-2.57 (m, 1H, H-4), 2.71-2.79 (m, 1H, H-4), 3.55 (qui, $J=6.9 \mathrm{~Hz}, 1 \mathrm{H}, H-6$ ), 3.91 (broad, $1 \mathrm{H}$, $H-9$ ), 5.28 (s, 1H, H-7), 6.25 (s, 1H, H-7); ${ }^{13} \mathrm{C}$ NMR (125 MHz, CDCl $\left.{ }_{3}\right) \delta 11.56(C-11), 20.31(C-8)$, 24.10 (C-5 or 12), 25.38 (C-5 or 12), 26.17 (C-5 or 12), 29.55 (C-4), 49.97 (C-6), 61.05 (C-9), 121.89 $(C-7), 137.70(C-3), 164.66$ (C-2); IR (neat) 1655, 1609; MS (EI) $\mathrm{m} / z$ (rel intensity) $196\left(\mathrm{M}^{+}, 23\right), 195$ (100), 180 (21), 167 (39), 166 (100), 152 (11), 126 (11), 124 (5), 110 (7); HRMS (EI) m/z calcd for $\mathrm{C}_{12} \mathrm{H}_{21} \mathrm{NO}\left(\mathrm{M}^{+}\right)$195.1623, found 195.1614.

1-(1-Cyclohexylethyl)-6-methyl-3-methylene-piperidin-2-one (2f). 


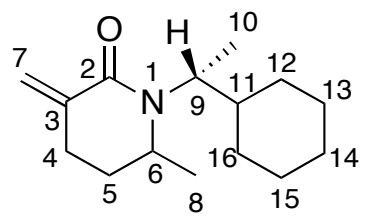

$\left(\mathrm{R}_{\mathrm{f}}=0.3\right.$, hexane/EtOAc $\left.=5 / 1\right)$. Obtained as a diastereomer mixture. ${ }^{1} \mathrm{H}$ NMR $\left(500 \mathrm{MHz}, \mathrm{CDCl}_{3}\right) \delta$ 0.73-0.93 (m, 4H,), 1.04-1.24 (m, 16H), $1.31(\mathrm{~d}, J=6.9 \mathrm{~Hz}, 3 \mathrm{H}), 1.58-1.94(\mathrm{~m}, 15 \mathrm{H}), 2.45-2.55(\mathrm{~m}, 2 \mathrm{H}$, $H-4), 2.60-2.76$ (m, 2H, $H-4$ ), 3.14 (broad, $1 \mathrm{H}, H-9$ ), 3.47 (t, $J=6.9 \mathrm{~Hz}, 1 \mathrm{H}, H-6$ ), 3.55 (t, $J=6.4 \mathrm{~Hz}$, $1 \mathrm{H}, H-6$ ), 4.08 (broad, $1 \mathrm{H}, H-9$ ), 5.19 (d, $J=0.9 \mathrm{~Hz}, 1 \mathrm{H}, H-7$ ), 5.27 (d, $J=1.4 \mathrm{~Hz}, 1 \mathrm{H}, H-7), 6.15$ (s, $1 \mathrm{H}, H-7), 6.21$ (s, $1 \mathrm{H}, H-7) ;{ }^{13} \mathrm{C}$ NMR $\left(125 \mathrm{MHz}, \mathrm{CDCl}_{3}\right) \delta 16.85,16.90,19.46,19.51,20.80,20.85$, $25.99,26.13,26.26,26.38,30.53,30.62,30.70,31.02,40.49,40.79,48.69,54.00,56.37,61.91,121.28$ (C-7), $122.12(C-7), 137.54(C-3), 138.12(C-3), 163.73(C-2), 164.41(C-2)$; IR (neat) 1649, 1609; MS (EI) $\mathrm{m} / \mathrm{z}$ (rel intensity) $235\left(\mathrm{M}^{+}, 8\right), 153$ (13), 152 (100), 126 (27), 83 (11), 81 (20), 70 (10), 67 (14), 55 (62), 54 (13), 53 (17); HRMS (EI) $m / z$ calcd for $\mathrm{C}_{15} \mathrm{H}_{25} \mathrm{NO}\left(\mathrm{M}^{+}\right)$235.1936, found 235.1939.

6-Methyl-3-methylene-1-(1-phenylethyl)-piperidine-2-one (2g).

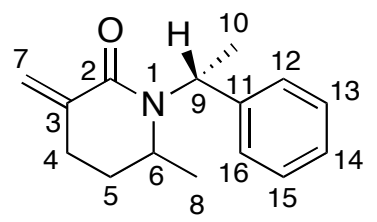

$\left(\mathrm{R}_{\mathrm{f}}=0.13\right.$, hexane/EtOAc $\left.=5 / 1\right)$. Obtained as a diasteromer mixture ${ }^{1} \mathrm{H}$ NMR $\left(500 \mathrm{MHz}, \mathrm{CDCl}_{3}\right) \delta$ $0.65(\mathrm{~d}, J=6.9 \mathrm{~Hz}, 3 \mathrm{H}, H-8), 1.24$ (d, $J=6.4 \mathrm{~Hz}, 3 \mathrm{H}, H-8), 1.46-1.62(\mathrm{~m}, 2 \mathrm{H}, H-5), 1.57$ (d, $J=7.3 \mathrm{~Hz}$, $3 \mathrm{H}, H-10), 1.61(\mathrm{~d}, J=6.8 \mathrm{~Hz}, 3 \mathrm{H}, H-10), 1.63-1.69(\mathrm{~m}, 1 \mathrm{H}, H-5), 1.83-1.90(\mathrm{~m}, 1 \mathrm{H}, H-5), 2.55-2.58$ (m, 2H, H-4), 2.69-2.81 (m, 2H, H-4), 3.34-3.37 (m, $1 \mathrm{H}, H-6), 3.66-3.69(\mathrm{~m}, 1 \mathrm{H}, H-6) 5.32(\mathrm{~d}, J=1.9$ $\mathrm{Hz}, 1 \mathrm{H}, H-7), 5.35(\mathrm{~d}, J=2.3 \mathrm{~Hz}, 1 \mathrm{H}, H-7), 5.94(\mathrm{q}, J=6.8 \mathrm{~Hz}, 1 \mathrm{H}, H-9), 6.07(\mathrm{q}, J=7.3 \mathrm{~Hz}, 1 \mathrm{H}, H-$ 9), $6.32(\mathrm{~s}, 1 \mathrm{H}, H-7), 6.34(\mathrm{~s}, 1 \mathrm{H}, H-7), 7.22-7.41(\mathrm{~m}, 10 \mathrm{H}, H-\mathrm{Ph}) ;{ }^{13} \mathrm{C}$ NMR $\left(125 \mathrm{MHz}, \mathrm{CDCl}_{3}\right) \delta$ 16.13 (C-10), $17.31(C-10), 19.71(C-8), 20.96(C-8), 24.05(C-4), 24.23(C-4), 29.33(C-5), 29.85(C-$ 5), $47.76(C-6), 48.63(C-6), 51.80(C-9), 52.11(C-9), 122.79(C-2), 123.07(C-2), 127.37(C-P h)$, $127.42(C-P h), 127.45(C-P h), 128.00(C-P h), 128.37(C-P h), 128.46(C-P h), 137.15(C-3), 137.41(C-$ 3), 140.83 (C-11), 141.02 (C-11), 164.13 (C-2), 164.52 (C-2),; IR (neat) 2930, 2970, 2850, 1655, 1610, 1450, 1430; MS (EI) $\mathrm{m} / \mathrm{z}$ (rel intensity) $229\left(\mathrm{M}^{+}, 83\right), 214$ (22), 172 (73), 105 (100); HRMS (EI) $\mathrm{m} / \mathrm{z}$ calcd for $\mathrm{C}_{15} \mathrm{H}_{19} \mathrm{NO}\left(\mathrm{M}^{+}\right), 229.1467$ found 229.1469 .

6-Methyl-3-methylene-1-(1,2,2-trimethylpropyl)-piperidin-2-one (2h).

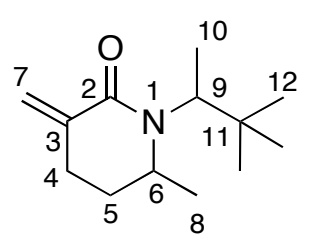

$\left(\mathrm{R}_{\mathrm{f}}=0.33\right.$, hexane/EtOAc $\left.=5 / 1\right)$. For major isomer; ${ }^{1} \mathrm{H}$ NMR $\left(500 \mathrm{MHz}, \mathrm{CDCl}_{3}\right) \delta 0.91(\mathrm{~s}, 9 \mathrm{H}, H-12)$, 1.17- 1.21 (m, 6H, H-8, 10), 1.66-1.71 (m, $1 \mathrm{H}, H-5), 1.94-2.02$ (m, $1 \mathrm{H}, H-5), 2.61-2.65$ (m, $1 \mathrm{H}, H-4)$, 
2.76-2.84 (m, 1H, H-4), 3.72-3.78 (m, 1H, H-O), 4.75-4.80 (m, 1H, H-9), 5.28-5.31 (m, 1H, H-7), 6.23$6.25(\mathrm{~m}, 1 \mathrm{H}, \mathrm{H}-7) ;{ }^{13} \mathrm{C}$ NMR $\left(125 \mathrm{MHz}, \mathrm{CDCl}_{3}\right) \delta 13.27$ (C-10), 21.09 (C-8), $23.32(C-4), 27.47(C-4)$, 28.00 (C-12), 36.61 (C-11), 47.78 (C-6), 55.38 (C-9), 123.15 (C-7), 137.20 (C-3), $165.55(C-2)$; IR (neat) 1651, 1610, 1428; MS (EI) $\mathrm{m} / z$ (rel intensity) $209\left(\mathrm{M}^{+}, 2\right), 152$ (100), 108 (14), 81 (11); HRMS (EI) $m / z$ calcd for $\mathrm{C}_{13} \mathrm{H}_{23} \mathrm{NO}\left(\mathrm{M}^{+}\right)$209.1780, found 209.1775. For minor isomer (Minor isomer was obtained as a mixture with major isomer.); ${ }^{1} \mathrm{H} \mathrm{NMR}\left(500 \mathrm{MHz}, \mathrm{CDCl}_{3}\right) \delta 0.99(\mathrm{~s}, 9 \mathrm{H}, \mathrm{H}-12), 1.23-1.25$ (m, $3 \mathrm{H}, H-8$ or 10$), 1.53(\mathrm{~d}, J=7.3 \mathrm{~Hz}, 3 \mathrm{H}, H-8$ or 10$), 1.60-1.71(\mathrm{~m}, 1 \mathrm{H}, H-5), 1.97-2.05(\mathrm{~m}, 1 \mathrm{H}, H-$ 5), 2.51-2.57 (m, 1H, H-4), 2.65-2.74 (m, 1H, H-4), 3.45-3.53 (m, 1H, H-6), 4.72-4.78 (m, 1H, H-9), $5.22(\mathrm{~s}, 1 \mathrm{H}, H-7), 6.20(\mathrm{~s}, 1 \mathrm{H}, H-7)$.

\section{1-Benzyl-8,8-dimethyl-6-methylene-hexahydroindolozin-5-one (2i).}

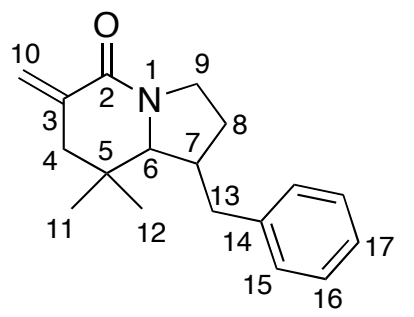

$\left(\mathrm{R}_{\mathrm{f}}=0.13\right.$, hexane/EtOAc $\left.=5 / 1\right)$.diasteromer mixture ${ }^{1} \mathrm{H}$ NMR $\left(500 \mathrm{MHz}, \mathrm{CDCl}_{3}\right) \delta 0.84(\mathrm{~s}, 3 \mathrm{H}, H-11$ or 12), 1.03 (s, 3H, H-11 or 12), 1.07 (s, 3H, H-11 or 12), 1.22 (s, 3H, H-11 or 12), 1.51-1.64 (m, $1 \mathrm{H}$, $H-7)$, 1.68-1.74 (m, 1H, H-7), 2.24-2.36 (m, 3H, H-4, 7, 9), 2.39-2.47 (m, 2H, H-4, 9), 2.61-2.66 (m, $1 \mathrm{H}, H-4), 2.99-3.05(\mathrm{~m}, 1 \mathrm{H}, H-4), 3.21(\mathrm{~d}, J=10.8 \mathrm{~Hz}, 1 \mathrm{H}, H-6), 3.42-3.47$ (m, $1 \mathrm{H}, H-11), 3.54-3.68$ $(\mathrm{m}, 2 \mathrm{H}, H-6,11), 5.25$ (d, $J=1.9 \mathrm{~Hz}, 1 \mathrm{H}, H-7), 5.25$ (s, 1H, H-10), $6.22(\mathrm{~s}, 1 \mathrm{H}, H-10), 7.10-7.30(\mathrm{~m}$, $10 \mathrm{H}, \mathrm{H}-\mathrm{Ph}) ;{ }^{13} \mathrm{C} \mathrm{NMR}\left(125 \mathrm{MHz}, \mathrm{CDCl}_{3}\right.$ ) $\delta 18.71$ (C-12 or 13), 21.16 (C-12 or 13), 26.22 (C-8), 26.69 (C-12 or 13), 27.53 (C-12 or 13), 29.60 (C-8), 33.63 (C-7), 34.01 (C-7), 40.31 (C-4), 42.77(C-4), 43.37 (C-11), 44.43 (C-11), 45.76 (C-9), 48.67 (C-9), 62.46 (C-5), 66.49 (C-5), $70.18(C-6), 71.54(C-6)$, 121.58 (C-10), 121.61 (C-10), 126.17 (C-Ph), 126.28 (C-Ph), $128.46(C-P h), 128.77(C-P h), 136.05$ (C-3), 136.92 (C-3), 139.70 (C-11), 140.00 (C-11), 162.91 (C-2), 163.66 (C-2); IR (neat) 3027, 2957, 1656, 1616, 1496, 1452; MS (EI) $m / z$ (rel intensity) $269\left(\mathrm{M}^{+}, 5\right), 178$ (83), 152 (10), 84 (100); HRMS (EI) $m / z$ calcd for $\mathrm{C}_{18} \mathrm{H}_{23} \mathrm{NO}\left(\mathrm{M}^{+}\right), 269.1780$ found 269.1782 .

\section{1-(1-Ethylpropyl)-5,5-dimethyl-5,6-dihydro-1H-pyridin-2-one (2j).}<smiles>[3H]C1(C)C=CC(=O)N(C(CC)CC)C1</smiles>

${ }^{1} \mathrm{H}$ NMR (500 MHz, $\left.\mathrm{CDCl}_{3}\right) \delta 0.88$ (t, $\left.J=7.3 \mathrm{~Hz} 6 \mathrm{H}, \mathrm{H}-10\right), 1.11$ (s, 6H, H-7), 1.35-1.53 (m, 4H, H-9), 2.95 (s, 2H, H-6), $4.42(\mathrm{sex}, J=5.0 \mathrm{~Hz}, 2 \mathrm{H}, H-6), 5.81(\mathrm{~d}, J=9.6 \mathrm{~Hz}, 1 \mathrm{H}, H-4), 6.23(\mathrm{~d}, J=9.6 \mathrm{~Hz}$, $1 \mathrm{H}, \mathrm{H}-3) ;{ }^{13} \mathrm{C}$ NMR (125 MHz, $\left.\mathrm{CDCl}_{3}\right) \delta 11.20$ (C-10) 24.83 (C-7 or 9), 26.11 (C-7 or 9), $32.52(C-6)$, $51.09(C-8), 55.02(C-5), 123.24(C-4), 148.71(C-3), 164.44(C-2)$; IR (neat) 1659, 1608, 1476, 1430; MS (EI) $m / z$ (rel intensity) $195\left(\mathrm{M}^{+}, 9\right), 166(100), 83(23)$; HRMS (EI) $m / z$ calcd for $\mathrm{C}_{12} \mathrm{H}_{21} \mathrm{NO}\left(\mathrm{M}^{+}\right)$, 195.1623 found 195.1614 . 
5-Oxo-3,3-dimethyl-6-methylene-2,7,8,8a-hexahydro-5H-oxazolo[3,2-a]pyridine (2k).

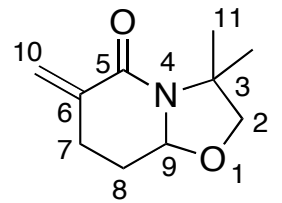

$\left(\mathrm{R}_{\mathrm{f}}=0.13\right.$, hexane/EtOAc $\left.=5 / 1\right) .{ }^{1} \mathrm{H}$ NMR $\left(500 \mathrm{MHz}, \mathrm{CDCl}_{3}\right) \delta 1.55(\mathrm{~d}, J=3.67 \mathrm{~Hz}, 6 \mathrm{H}, H-11), 1.57-$ $1.68(\mathrm{~m}, 1 \mathrm{H}, H-8), 2.27(\mathrm{dq}, J=11.91,3.52 \mathrm{~Hz}, 1 \mathrm{H}, H-8), 2.41-2.49$ (m, 1H, $H-7), 2.71$ (dt, $J=16.50$, $3.67 \mathrm{~Hz}, 1 \mathrm{H}, H-7), 3.62(\mathrm{~d}, J=8.71 \mathrm{~Hz}, 1 \mathrm{H}, H-2), 3.88(\mathrm{~d}, J=8.71 \mathrm{~Hz}, 1 \mathrm{H}, H-2), 4.82(\mathrm{dd}, J=10.08$, $3.21 \mathrm{~Hz}, 1 \mathrm{H}, H-9), 5.32(\mathrm{~s}, 1 \mathrm{H}, H-10), 6.24(\mathrm{t}, J=2.06 \mathrm{~Hz}, 1 \mathrm{H}, H-10) ;{ }^{13} \mathrm{C} \mathrm{NMR}\left(125 \mathrm{MHz}, \mathrm{CDCl}_{3}\right) \delta$ 23.42 (C-11), 24.59 (C-11), $25.74(C-7), 29.00(C-8), 60.30(C-3), 79.36(C-2), 88.90(C-9), 121.57(C$ 10), 137.10 (C-6), 161.31 (C-5); IR (neat) 1663, 1616; MS (EI) $\mathrm{m} / z$ (rel intensity) $181\left(\mathrm{M}^{+}, 74\right), 180$ (49), 166 (100), 149 (28), 138 (30), 77 (24), 54 (25); HRMS (EI) $m / z$ calcd for $\mathrm{C}_{10} \mathrm{H}_{15} \mathrm{NO}_{2}\left(\mathrm{M}^{+}\right)$ 181.1103, found 181.1102 .

(3S,8aR)-5-Oxo-3-isopropyl-6-methylene-2,3,7,8,8a-hexahydro-5H-oxazolo[3,2-a]pyridine (2l).<smiles>COC1COC2CCC(=O)C(=O)N12</smiles>

$\left(\mathrm{R}_{\mathrm{f}}=0.33\right.$, hexane/EtOAc $\left.=2 / 1\right) .{ }^{1} \mathrm{H}$ NMR $\left(500 \mathrm{MHz}, \mathrm{CDCl}_{3}\right) \delta 0.90(\mathrm{~d}, J=6.9 \mathrm{~Hz}, 3 \mathrm{H}, H-12$ or 13$)$, $0.92(\mathrm{~d}, J=6.9 \mathrm{~Hz}, 3 \mathrm{H}, H-12$ or 13$), 1.48-1.56(\mathrm{~m}, 1 \mathrm{H}, H-8), 2.16-2.32(\mathrm{~m}, 2 \mathrm{H}, H-8,11), 2.40-2.49$ (m, $1 \mathrm{H}, H-7), 2.65(\mathrm{dt}, J=15.6,3.7 \mathrm{~Hz}, 1 \mathrm{H}, H-7), 3.73(\mathrm{dd}, J=8.7,6.9 \mathrm{~Hz}, 1 \mathrm{H}, H-2), 4.07$ (t-like, $J=8.3$ $\mathrm{Hz}, 1 \mathrm{H}, H-2), 4.25(\mathrm{q}, J=6.9 \mathrm{~Hz}, 1 \mathrm{H}, H-3), 4.84(\mathrm{dd}, J=9.6,4.6 \mathrm{~Hz}, 1 \mathrm{H}, H-9), 5.36(\mathrm{~s}, 1 \mathrm{H}, H-10)$, $6.24(\mathrm{~s}, 1 \mathrm{H}, \mathrm{H}-10) ;{ }^{13} \mathrm{C}$ NMR $\left(125 \mathrm{MHz}, \mathrm{CDCl}_{3}\right) \delta 17.00$ (C-12 or 13), 19.03 (C-12 or 13), $25.44(C-4)$, $28.78(C-5$ or 11$), 29.76(C-5$ or 11$), 59.63(C-3), 66.87(C-2), 88.06(C-6), 121.59(C-10), 136.73(C-$ 6), 162.60 (C-5); IR (neat) 1664, 1620, 1445; MS (EI) m/z (rel intensity) 195 (M, 23), 152 (100), 124 (54); HRMS (EI) $m / z$ calcd for $\mathrm{C}_{11} \mathrm{H}_{17} \mathrm{NO}\left(\mathrm{M}^{+}\right)$195.1259, found 195.1260 .

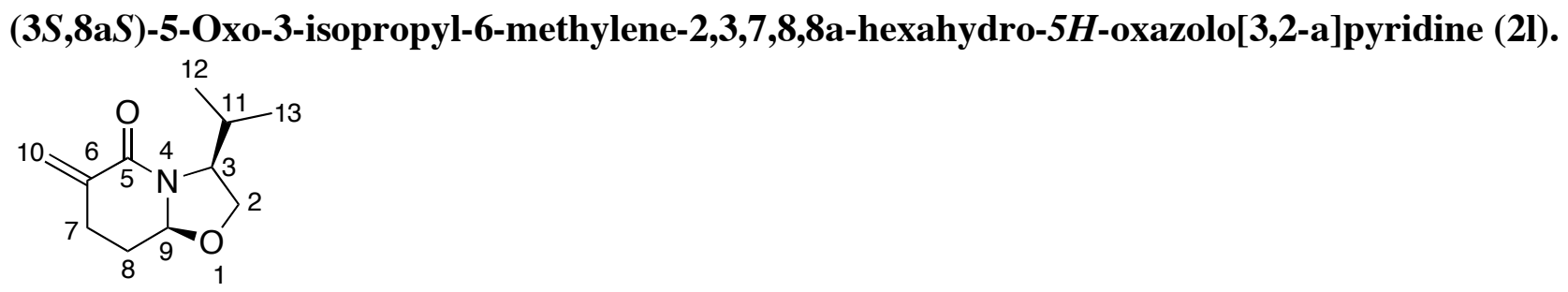

$\left(\mathrm{R}_{\mathrm{f}}=0.33\right.$, hexane/EtOAc $\left.=2 / 1\right) .{ }^{1} \mathrm{H} \mathrm{NMR}\left(500 \mathrm{MHz}, \mathrm{CDCl}_{3}\right) \delta 0.76(\mathrm{~d}, J=6.9 \mathrm{~Hz}, 3 \mathrm{H}, H-12$ or 13$)$, $0.91(\mathrm{~d}, J=6.9 \mathrm{~Hz}, 3 \mathrm{H}, H-12$ or 13$), 1.54-1.73(\mathrm{~m}, 1 \mathrm{H}, H-11), 2.24-2.27(\mathrm{~m}, 1 \mathrm{H}, H-8), 2.46-2.52(\mathrm{~m}$, $1 \mathrm{H}, H-8), 2.46-2.52(\mathrm{~m}, 1 \mathrm{H}, H-7), 2.75-2.86(\mathrm{~m}, 1 \mathrm{H}, H-7), 3.80(\mathrm{dd}, J=9.4,7.1 \mathrm{~Hz}, 1 \mathrm{H}, H-2), 4.00-$ $4.07(\mathrm{~m}, 1 \mathrm{H}, H-2), 4.07-4.10(\mathrm{~m}, 1 \mathrm{H}, H-3), 4.75$ (dd, $J=10.3,3.0 \mathrm{~Hz}, 1 \mathrm{H}, H-9), 5.37(\mathrm{~s}, 1 \mathrm{H}, H-10)$, 6.27 (s, 1H, H-10); IR (neat) 1655, 1459; MS (EI) $\mathrm{m} / z$ (rel intensity) 195 (M, 29), 152 (100), 124 (57); HRMS (EI) $m / z$ calcd for $\mathrm{C}_{11} \mathrm{H}_{17} \mathrm{NO}\left(\mathrm{M}^{+}\right)$195.1259, found 195.1250. 


\section{Synthesis of the precursor of (-)coniine.}

A magnetic stirring bar, AIBN (28.8 mg, $0.18 \mathrm{mmol})$, benzene $(20 \mathrm{~mL})$, oxazoline $1 \mathrm{~m}(173.1 \mathrm{mg}, 0.50$ $\mathrm{mmol})$ and $(\mathrm{TMS})_{3} \mathrm{SiH}(189 \mathrm{mg}, 0.77 \mathrm{mmol})$ were placed in a $50 \mathrm{~mL}$ stainless steel autoclave. The autoclave was closed, purged three times with carbon monoxide, pressurized with 71 atm of $\mathrm{CO}$ and then heated $90{ }^{\circ} \mathrm{C}$ for $10 \mathrm{~h}$. Excess $\mathrm{CO}$ was discharged at room temperature. The solvent was removed under reduced pressure. The residue was purified by flash chromatography on silica gel (gradient from hexane/EtOAc $=1 / 1$ to EtOAc) to give cis isomer $\mathbf{2 m}(53.4 \mathrm{mg}, 49 \%)$. Trans isomer was farther purified by preparative HPLC (17.4 mg, 16\%).

(3R,8aR)-5-Oxo-3-phenyl-2,3,7,8,8a-hexahydro-5H-oxazolo[3,2-a]pyridine (2m).

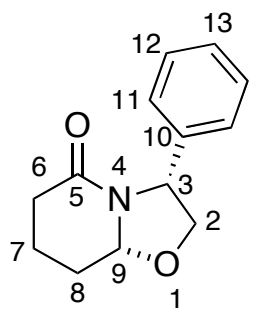

$\left(\mathrm{R}_{\mathrm{f}}=0.17\right.$, EtOAc). ${ }^{1} \mathrm{H}$ NMR $\left(500 \mathrm{MHz}, \mathrm{CDCl}_{3}\right) \delta$ 1.72-1.80 (m, 2H, H-7 or 8) 2.01-2.06 (m, $1 \mathrm{H}, H-7$ or 8$), 2.24-2.31(\mathrm{~m}, 1 \mathrm{H}, H-6$ or 7 or 8$), 2.35-2.41(\mathrm{~m}, 2 \mathrm{H}, H-6,7$ or 8$), 4.01(\mathrm{dd}, J=8.9,1.1 \mathrm{~Hz}, 1 \mathrm{H}$, $H-2$ or 3$), 4.15(\mathrm{dd}, J=9.2,6.9 \mathrm{~Hz}, 1 \mathrm{H}, H-2$ or 3$), 4.85(\mathrm{dd}, J=9.4,3.4 \mathrm{~Hz}, 1 \mathrm{H}, H-2$ or 3$), 4.92(\mathrm{~d}, J=$ $6.9 \mathrm{~Hz}, 1 \mathrm{H}, H-9), 7.22-7.36(\mathrm{~m}, 5 \mathrm{H}, H-P h) ;{ }^{13} \mathrm{C} \mathrm{NMR}\left(100 \mathrm{MHz}, \mathrm{CDCl}_{3}\right) \delta 17.78(C-7), 28.20(C-8)$, $30.99(C-6), 58.74(C-3), 73.78(C-2), 88.86(C-9), 126.32(C-11), 127.45(C-13), 128.50(C-12)$, $141.47(C-10), 167.31(C-2)$; This product is a known compound and was identified by NMR with comparison of literature data (Amat, M. et al. J. Org. Chem. 2000, 65, 3074.).

(3R,8aS)-5-Oxo-3-phenyl-2,3,7,8,8a-hexahydro-5H-oxazolo[3,2-a]pyridine (2m).

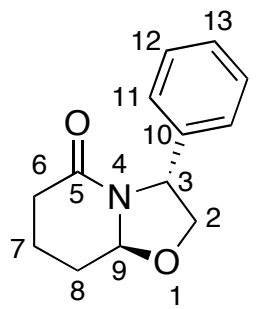

$\left(\mathrm{R}_{\mathrm{f}}=0.1\right.$, hexane/EtOAc $\left.=1 / 2\right) .{ }^{1} \mathrm{H}$ NMR $\left(500 \mathrm{MHz}, \mathrm{CDCl}_{3}\right) \delta 151-1.60(\mathrm{~m}, 1 \mathrm{H}, H-7$ or 8$), 1.73-1.83$ $(\mathrm{m}, 1 \mathrm{H}, H-7$ or 8$), 1.95-2.01(\mathrm{~m}, 1 \mathrm{H}, H-7$ or 8$), 2.31-2.40(\mathrm{~m}, 2 \mathrm{H}, H-6,7$ or 8$), 2.53(\mathrm{dd}, J=17.9,6.0$ $\mathrm{Hz}, 1 \mathrm{H}, H-6), 3.77(\mathrm{dd}, J=9.2,7.8 \mathrm{~Hz}, 1 \mathrm{H}, H-2$ or 3$), 4.48-4.51(\mathrm{~m}, 1 \mathrm{H}, H-2$ or 3$), 5.03(\mathrm{dd}, J=9.2$, $4.6 \mathrm{~Hz}, 1 \mathrm{H}, H-2$ or 3$), 5.28(\mathrm{t}, J=7.8 \mathrm{~Hz}, 1 \mathrm{H}, H-9), 7.25-7.36(\mathrm{~m}, 5 \mathrm{H}, H-P h) ;{ }^{13} \mathrm{C} \mathrm{NMR}(100 \mathrm{MHz}$, $\left.\mathrm{CDCl}_{3}\right) \delta 17.16(C-7), 28.48(C-8), 31.38(C-6), 58.08$ (C-3), 72.45 (C-2), 88.67 (C-9), $126.09(C-11)$, $127.59(C-13), 128.80(C-12), 139.62(C-10), 168.87(C-2)$ This product is a known compound and was identified by NMR with comparison of literature data (Amat, M. et al. J. Org. Chem. 2000, 65, 3074.). 


\section{Computational Chemistry}

All density functional (DFT) calculations were carried out on a DACS XJ-3000 computer using the Gaussian 98 program. ${ }^{1}$ Geometry optimizations were performed using standard gradient techniques ${ }^{2}$ at the BHandHLYP level of theory. All calculations were performed using standard basis sets. BHandHLYP/cc-pVDZ Gaussian Archive entries and imaginary frequencies for the optimized structures in this study and BHandHLYP/aug-cc-pVDZ//BHandHLYP/cc-pVDZ calculated singlepoint energies are listed below.

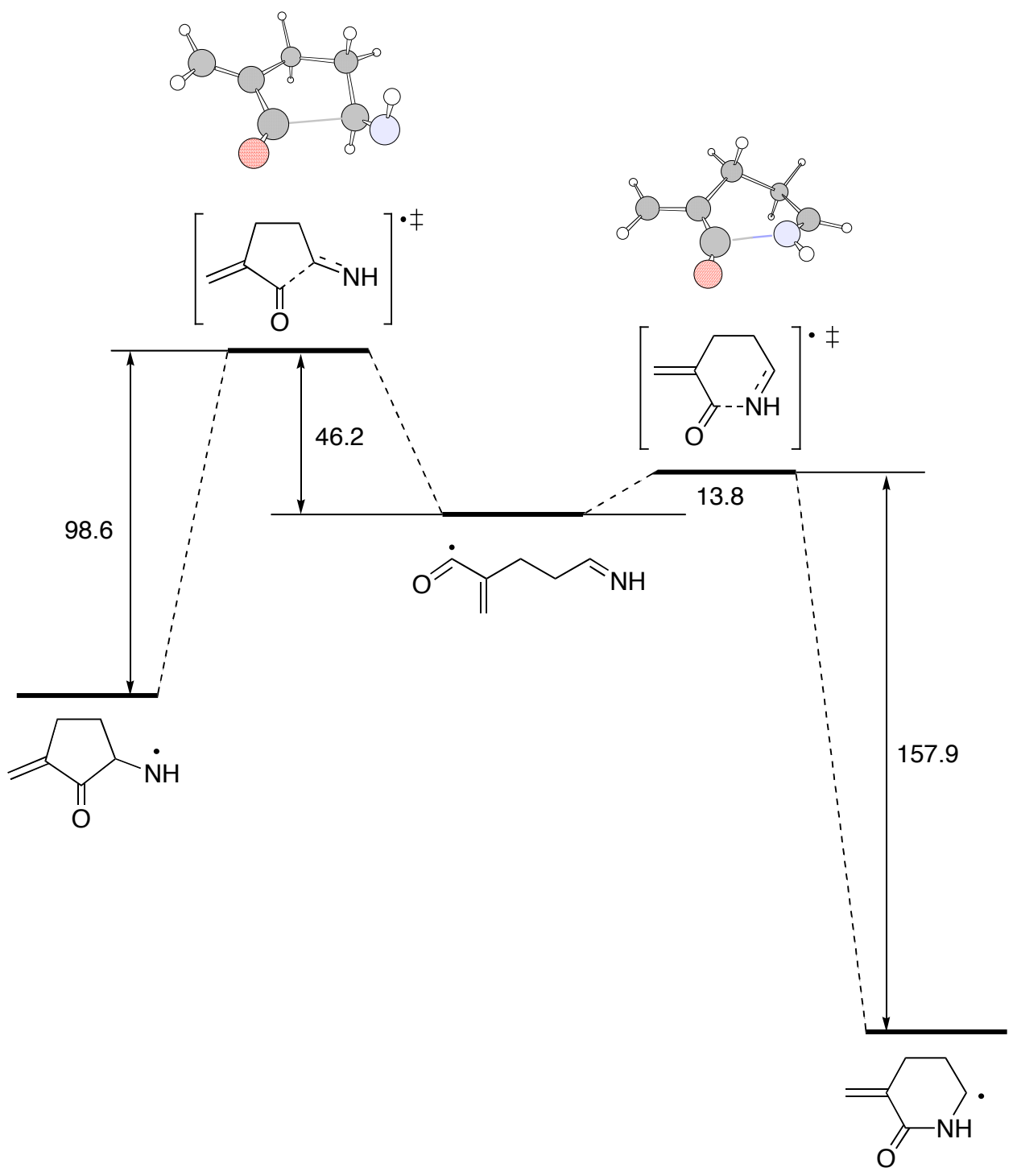

Figure S1. Reaction profile of 5-exo and 6-endo cyclization of 2-methylene-6-aza-5-hexnenoyl radical. Energies (in $\mathrm{kJ} / \mathrm{mol}$ ) are calculated on the BHandHLYP/aug-cc-pVDZ//BHandHLYP/cc-pVDZ. 


\section{Transition state involved in the 6-endo cyclization BHandHLYP/cc-pVDZ}

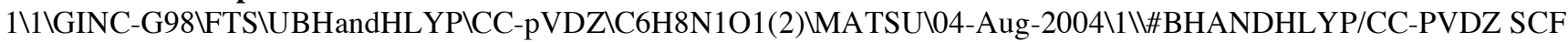
$=$ DIRECT FREQ=NORAMAN GEOM=CHECK GUESS=READ OPT=(TS,NOEIGENTEST,Z-MATRIX,READFC,MAX CYCLE=100) NOSYMM \TS for 6-endo cyclization $\backslash \backslash 0,2 \backslash C \backslash N, 1, \mathrm{r} 1 \backslash \mathrm{C}, 2, \mathrm{r} 2,1, \mathrm{a} 1 \backslash \mathrm{C}, 3, \mathrm{r} 3,2, \mathrm{a} 2,1, \mathrm{~d} 1,0 \backslash \mathrm{C}, 4, \mathrm{r} 4,3, \mathrm{a} 3,2, \mathrm{~d} 2,0 \backslash \mathrm{C}, 1, \mathrm{r} 5$,

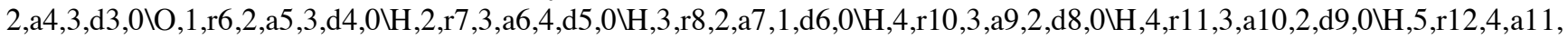
$3, \mathrm{~d} 10,0 \backslash \mathrm{H}, 5, \mathrm{r} 13,4, \mathrm{a} 12,3, \mathrm{~d} 11,0 \backslash \mathrm{C}, 6, \mathrm{r} 14,5, \mathrm{a} 13,4, \mathrm{~d} 12,0 \backslash \mathrm{H}, 14, \mathrm{r} 15,6, \mathrm{a} 14,1, \mathrm{~d} 13,0 \backslash \mathrm{H}, 14, \mathrm{r} 16,6, \mathrm{a} 15,1, \mathrm{~d} 14,0 \backslash \mathrm{r} 1=1.97882429 \backslash \mathrm{r} 2=1.2$ $6149624 \backslash \mathrm{r} 3=1.48926628 \backslash \mathrm{r} 4=1.52301771 \backslash \mathrm{r} 5=1.3703798 \backslash \mathrm{r} 6=1.17943117 \mathrm{r} 7=1.01436957 \backslash \mathrm{r} 8=1.09400737 \mathrm{r} 10=1.09524173 \backslash \mathrm{r} 1$ $1=1.09917451 \backslash \mathrm{r} 12=1.09686563 \backslash \mathrm{r} 13=1.09140959 \backslash \mathrm{r} 14=1.40269171 \backslash \mathrm{r} 15=1.08186648 \backslash \mathrm{r} 16=1.0824963 \backslash \mathrm{a} 1=122.05211881 \backslash \mathrm{a} 2=1$ $18.20420266 \backslash \mathrm{a} 3=111.36213781 \backslash \mathrm{a} 4=102.50267173 \backslash \mathrm{a} 5=105.84412235 \backslash \mathrm{a} 6=117.08467117 \backslash \mathrm{a} 7=122.28658887 \backslash \mathrm{a} 9=109.178731$ $05 \backslash \mathrm{a} 10=107.45743995 \backslash \mathrm{a} 11=108.05284133 \backslash \mathrm{a} 12=108.69058759 \backslash \mathrm{a} 13=120.11712423 \backslash \mathrm{a} 14=121.34583792 \backslash \mathrm{a} 15=119.98129928 \backslash$ $\mathrm{d} 1=23.16482539 \backslash \mathrm{d} 2=17.52848836 \backslash \mathrm{d} 3=-29.1693359 \backslash \mathrm{d} 4=-211.21004475 \backslash \mathrm{d} 5=176.68240468 \backslash \mathrm{d} 6=204.63123962 \backslash \mathrm{d} 8=140.5412$ $2141 \backslash \mathrm{d} 9=-104.56647447 \backslash \mathrm{d} 10=66.56305262 \backslash \mathrm{d} 11=181.69080994 \backslash \mathrm{d} 12=-131.83215814 \backslash \mathrm{d} 13=-0.08018392 \backslash \mathrm{d} 14=179.62172293 \backslash$ IVersion $=x 86-$ Linux-G98RevA.9 $\backslash H F=-363.1274887 \backslash \mathrm{S} 2=0.78771 \backslash \mathrm{S} 2-1=0 . \mathrm{S} 2 \mathrm{~A}=0.75033 \backslash \mathrm{RMSD}=9.170 \mathrm{e}-09 \backslash \mathrm{RMSF}=1.034 \mathrm{e}-$ 05\ipole=0.8298419,0.1159162,2.021128\PG=C01 [X(C6H8N1O1)]\@

Imaginary frequency: $-287.4418 \mathrm{~cm}^{-1}$.

BHandHLYP/aug-cc-pVDZ//BHandHLYP/cc-pVDZ $\mathrm{HF}=-363.1514607$

\section{Transition state involved in the 5-exo cyclization BHandHLYP/cc-pVDZ}

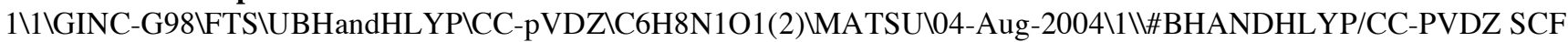
$=$ DIRECT FREQ=NORAMAN GEOM=CHECK GUESS=READ OPT=(TS,NOEIGENTEST,Z-MATRIX,READFC,MAX CYCLE=100) NOSYMM $\backslash T S$ for 5-exo cyclization $\backslash \backslash 0,2 \backslash O \backslash C, 1, \mathrm{r} 2 \backslash \mathrm{C}, 2, \mathrm{r} 3,1, \mathrm{a} 2 \backslash \mathrm{C}, 3, \mathrm{r} 4,2, \mathrm{a} 3,1, \mathrm{~d} 2,0 \backslash \mathrm{C}, 2, \mathrm{r} 5,3, \mathrm{a} 4,4, \mathrm{~d} 3,0 \backslash \mathrm{C}, 3, \mathrm{r} 6,2$,

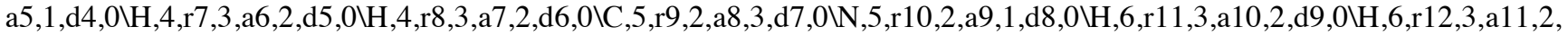
$\mathrm{d} 10,0 \backslash \mathrm{H}, 9, \mathrm{r} 13,5, \mathrm{a} 12,2, \mathrm{~d} 11,0 \backslash \mathrm{H}, 9, \mathrm{r} 14,5, \mathrm{a} 13,2, \mathrm{~d} 12,0 \backslash \mathrm{H}, 5, \mathrm{r} 15,2, \mathrm{a} 14,1, \mathrm{~d} 13,0 \backslash \mathrm{H}, 10, \mathrm{r} 16,5, \mathrm{a} 15,2, \mathrm{~d} 14,0 \backslash \mathrm{r} 2=1.17200165 \backslash \mathrm{r} 3=1.485$ $55496 \backslash \mathrm{r} 4=1.32885288 \backslash \mathrm{r} 5=2.15634876 \backslash \mathrm{r} 6=1.49724536 \backslash \mathrm{r} 7=1.08459367 \mathrm{r} 8=1.08576552 \backslash \mathrm{r} 9=1.51469248 \backslash \mathrm{r} 10=1.30089783 \backslash \mathrm{r} 11$ $=1.09168634 \backslash \mathrm{r} 12=1.09738928 \backslash \mathrm{r} 13=1.09501398 \backslash \mathrm{r} 14=1.09603986 \backslash \mathrm{r} 15=1.09569512 \backslash \mathrm{r} 16=1.02114858 \backslash \mathrm{a} 2=131.44449002 \backslash \mathrm{a} 3=1$ $19.42682209 \backslash \mathrm{a} 4=98.88546252 \backslash \mathrm{a} 5=112.10575635 \backslash \mathrm{a} 6=120.19728616 \backslash \mathrm{a} 7=121.76355403 \backslash \mathrm{a} 8=90.97756483 \backslash \mathrm{a} 9=108.55191618 \backslash$ a $10=111.53212626 \backslash \mathrm{a} 11=109.15689121 \backslash \mathrm{a} 12=109.12561244 \backslash \mathrm{a} 13=108.97573494 \backslash \mathrm{a} 14=90.12970196 \backslash \mathrm{a} 15=109.44405919 \backslash \mathrm{d} 2=7$ $.61679803 \backslash \mathrm{d} 3=-169.22500056 \backslash \mathrm{d} 4=-174.6994397 \backslash \mathrm{d} 5=-2.01437046 \backslash \mathrm{d} 6=177.82518814 \backslash \mathrm{d} 7=16.75857567 \backslash \mathrm{d} 8=-31.68971986 \backslash \mathrm{d}$ $9=-156.090153 \backslash \mathrm{d} 10=86.00912363 \backslash \mathrm{d} 11=-157.63919679 \backslash \mathrm{d} 12=85.51506156 \backslash \mathrm{d} 13=85.27255209 \backslash \mathrm{d} 14=-89.18042924 \| \mathrm{Version}=$

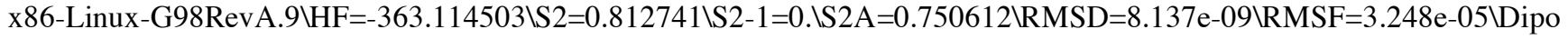
le=1.3974668,0.070167,1.3132957/PG=C01 [X(C6H8N1O1)]\@

Imaginary frequency: $-383.1360 \mathrm{~cm}^{-1}$.

BHandHLYP/aug-cc-pVDZ//BHandHLYP/cc-pVDZ $\mathrm{HF}=-363.1391292$

\section{2-Methylene-5-aza-5-hexenoyl radical BHandHLYP/cc-pVDZ}

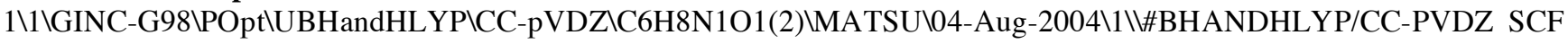
$=$ DIRECT FREQ=NORAMAN GEOM=CHECK GUESS=READ OPT=(Z-MATRIX,READFC,MAXCYCLE=100) NOS

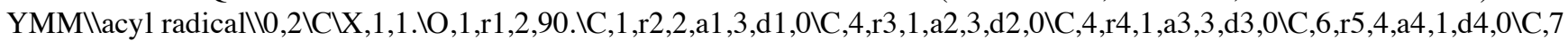

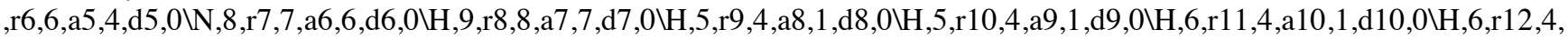

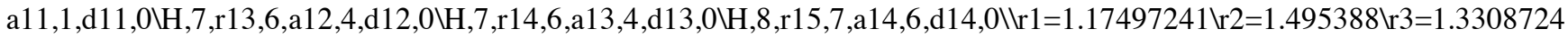
$3 \backslash \mathrm{r} 4=1.49813274 \backslash \mathrm{r} 5=1.53189597 \backslash \mathrm{r} 6=1.49646858 \backslash \mathrm{r} 7=1.26051555 \backslash \mathrm{r} 8=1.01820478 \backslash \mathrm{r} 9=1.08414637 \mathrm{r} 10=1.08591666 \backslash \mathrm{r} 11=1.0$ $9437239 \backslash \mathrm{r} 12=1.09580212 \backslash \mathrm{r} 13=1.09281309 \backslash \mathrm{r} 14=1.09709962 \backslash \mathrm{r} 15=1.10042025 \backslash \mathrm{a} 1=39.17331708 \backslash \mathrm{a} 2=117.79042773 \backslash \mathrm{a} 3=116.7$ $3910276 \backslash a 4=112.57838981 \backslash a 5=112.08008224 \backslash a 6=121.56699612 \backslash a 7=110.54608986 \backslash a 8=120.43592986 \backslash a 9=121.51811854 \backslash a$ $10=109.15730962 \backslash \mathrm{a} 11=108.98997774 \backslash \mathrm{a} 12=110.56818607 \backslash \mathrm{a} 13=108.90692489 \backslash \mathrm{a} 14=115.33392017 \backslash \mathrm{d} 1=171.77418693 \backslash \mathrm{d} 2=-4$ $.9081405 \backslash \mathrm{d} 3=175.9191716 \backslash \mathrm{d} 4=-65.88867675 \backslash \mathrm{d} 5=180.39161648 \backslash \mathrm{d} 6=241.22339868 \backslash \mathrm{d} 7=179.72351888 \backslash \mathrm{d} 8=1.06232786 \backslash \mathrm{d} 9=$ 
$181.44573431 \backslash \mathrm{d} 10=-188.59817824 \backslash \mathrm{d} 11=54.71154187 \backslash \mathrm{d} 12=59.60473482 \backslash \mathrm{d} 13=-58.95788428 \backslash \mathrm{d} 14=60.6619819 \backslash \backslash$ Version $=x 8$

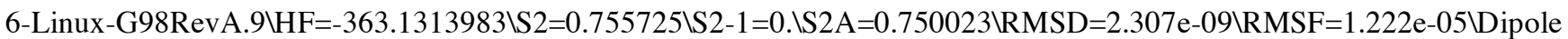
$=-1.0244851,-0.2327926,1.316042 \mathrm{PG}=\mathrm{C} 01[\mathrm{X}(\mathrm{C} 6 \mathrm{H} 8 \mathrm{~N} 1 \mathrm{O} 1)] \backslash @$

\section{BHandHLYP/aug-cc-pVDZ//BHandHLYP/cc-pVDZ} $\mathrm{HF}=-363.1567105$

\section{2-Aza-4-methylene-3-oxocyclohexyl radical (product of the 6-endo cyclization) BHandHLYP/cc-pVDZ}

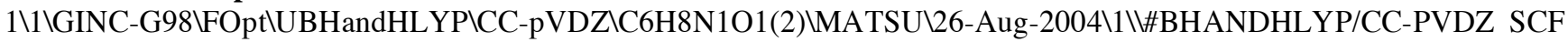
$=$ DIRECT FREQ=NORAMAN GEOM=CHECK GUESS=READ OPT=(Z-MATRIX,READFC,MAXCYCLE=100) NOS YMM \product of 6-endo cyclization $\backslash \backslash 0,2 \backslash \mathrm{C} \backslash \mathrm{N}, 1, \mathrm{r} 1 \backslash \mathrm{C}, 2, \mathrm{r} 2,1, \mathrm{a} 1 \backslash \mathrm{C}, 3, \mathrm{r} 3,2, \mathrm{a} 2,1, \mathrm{~d} 1,0 \backslash \mathrm{C}, 4, \mathrm{r} 4,3, \mathrm{a} 3,2, \mathrm{~d} 2,0 \backslash \mathrm{C}, 1, \mathrm{r} 5,2, \mathrm{a} 4,3, \mathrm{~d} 3,0 \backslash \mathrm{O}, 1$,

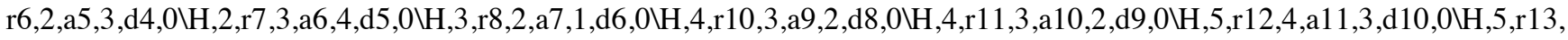
$4, \mathrm{a} 12,3, \mathrm{~d} 11,0 \backslash \mathrm{C}, 6, \mathrm{r} 14,5, \mathrm{a} 13,4, \mathrm{~d} 12,0 \backslash \mathrm{H}, 14, \mathrm{r} 15,6, \mathrm{a} 14,1, \mathrm{~d} 13,0 \backslash \mathrm{H}, 14, \mathrm{r} 16,6, \mathrm{a} 15,1, \mathrm{~d} 14,0 \backslash \backslash \mathrm{r} 1=1.37010576 \backslash \mathrm{r} 2=1.38350123 \backslash \mathrm{r} 3=1.4$ $85449 \backslash \mathrm{r} 4=1.52648282 \backslash \mathrm{r} 5=1.49474885 \backslash \mathrm{r} 6=1.21184022 \backslash \mathrm{r} 7=1.00659195 \backslash \mathrm{r} 8=1.08197211 \backslash \mathrm{r} 10=1.0931298 \backslash \mathrm{r} 11=1.10334221 \backslash \mathrm{r} 12$ $=1.09745737 \backslash \mathrm{r} 13=1.09232953 \backslash \mathrm{r} 14=1.32799684 \backslash \mathrm{r} 15=1.08346818 \backslash \mathrm{r} 16=1.08498895 \backslash \mathrm{a} 1=127.11464757 \backslash \mathrm{a} 2=117.2669017 \backslash \mathrm{a} 3=$ $109.83043476 \backslash a 4=115.3901974 \backslash a 5=120.65518891 \backslash a 6=119.37292381 \backslash \mathrm{a} 7=115.7949156 \backslash \mathrm{a} 9=109.90942246 \backslash \mathrm{a} 10=110.574977$ $43 \backslash \mathrm{a} 11=108.43949898 \backslash \mathrm{a} 12=111.21475702 \backslash \mathrm{a} 13=124.6922384 \backslash \mathrm{a} 14=119.89246535 \backslash \mathrm{a} 15=121.42174472 \backslash \mathrm{d} 1=-11.98693557 \backslash \mathrm{d} 2$ $=39.93069688 \backslash \mathrm{d} 3=-2.82647069 \backslash \mathrm{d} 4=-182.99831036 \backslash \mathrm{d} 5=168.11728275 \backslash \mathrm{d} 6=190.81095775 \backslash \mathrm{d} 8=162.16424751 \backslash \mathrm{d} 9=-80.31957$ $722 \backslash \mathrm{d} 10=66.34810796 \backslash \mathrm{d} 11=183.95064073 \backslash \mathrm{d} 12=-139.57987246 \backslash \mathrm{d} 13=0.79464027 \backslash \mathrm{d} 14=180.44315459 \backslash \backslash$ Version=x 86 -Linux-

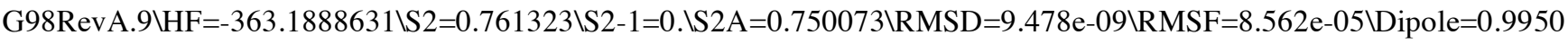
9,-0.0477085,0.8475058\PG=C01 [X(C6H8N1O1)]\@

\section{BHandHLYP/aug-cc-pVDZ//BHandHLYP/cc-pVDZ}

$\mathrm{HF}=-363.2116084$

\section{3-Methylene-2-oxocyclopentylaminyl radical (product of the 5-exo cyclization) BHandHLYP/cc-pVDZ}

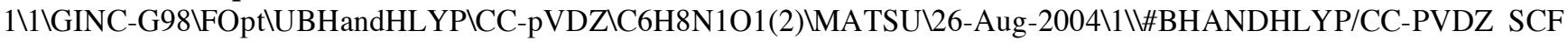
$=$ DIRECT FREQ=NORAMAN GEOM=CHECK GUESS=READ OPT=(Z-MATRIX,READFC,MAXCYCLE=100) NOS YMM \product of 5-exo cyclization $\backslash \backslash 0,2 \backslash \mathrm{O} \backslash \mathrm{C}, 1, \mathrm{r} 2 \backslash \mathrm{C}, 2, \mathrm{r} 3,1, \mathrm{a} 2 \backslash \mathrm{C}, 3, \mathrm{r} 4,2, \mathrm{a} 3,1, \mathrm{~d} 2,0 \backslash \mathrm{C}, 2, \mathrm{r} 5,3, \mathrm{a} 4,4, \mathrm{~d} 3,0 \backslash \mathrm{C}, 3, \mathrm{r} 6,2, \mathrm{a} 5,1, \mathrm{~d} 4,0 \backslash \mathrm{H}, 4, \mathrm{r}$

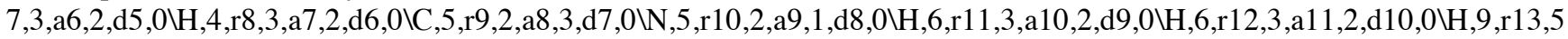

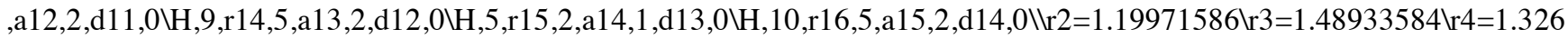
$82139 \backslash \mathrm{r} 5=1.52962744 \backslash \mathrm{r} 6=1.50674476 \backslash \mathrm{r} 7=1.08499349 \backslash \mathrm{r} 8=1.08495625 \backslash \mathrm{r} 9=1.52828811 \backslash \mathrm{r} 10=1.42539441 \backslash \mathrm{r} 11=1.09141743 \backslash \mathrm{r} 1$ $2=1.09697891 \backslash \mathrm{r} 13=1.09056821 \backslash \mathrm{r} 14=1.09451387 \backslash \mathrm{r} 15=1.10772633 \backslash \mathrm{r} 16=1.02619384 \backslash \mathrm{a} 2=127.6632467 \backslash \mathrm{a} 3=121.80899895 \backslash \mathrm{a} 4$ $=107.6367376 \backslash \mathrm{a} 5=108.09233062 \backslash \mathrm{a} 6=119.38212964 \backslash \mathrm{a} 7=122.35321506 \backslash \mathrm{a} 8=103.87517873 \backslash \mathrm{a} 9=115.07594247 \backslash \mathrm{a} 10=112.8883$ $6655 \backslash \mathrm{a} 11=109.62841842 \backslash \mathrm{a} 12=112.87521349 \backslash \mathrm{a} 13=108.21633894 \backslash \mathrm{a} 14=104.53380157 \backslash \mathrm{a} 15=105.51611752 \backslash \mathrm{d} 2=1.53956075 \backslash \mathrm{d}$ $3=-177.00213819 \backslash \mathrm{d} 4=-180.62290135 \backslash \mathrm{d} 5=-1.62774352 \backslash \mathrm{d} 6=178.09823293 \backslash \mathrm{d} 7=19.95474129 \backslash \mathrm{d} 8=-30.95133204 \backslash \mathrm{d} 9=-144.329$ $21595 \backslash \mathrm{d} 10=96.34259576 \backslash \mathrm{d} 11=-156.05918035 \backslash \mathrm{d} 12=84.80023957 \backslash \mathrm{d} 13=87.06401517 \backslash \mathrm{d} 14=35.56600321 \| V$ Version $=x 86-$ Linu $\mathrm{x}-\mathrm{G} 98 \mathrm{RevA} .9 \mathrm{VHF}=-363.1526232 \backslash \mathrm{S} 2=0.754949 \backslash \mathrm{S} 2-1=0 . \mathrm{SS} 2 \mathrm{~A}=0.750018 \backslash \mathrm{RMSD}=4.889 \mathrm{e}-09 \backslash \mathrm{RMSF}=1.133 \mathrm{e}-05 \backslash \mathrm{Dipole}=0.67$ $0358,-0.3865409,0.8966926 \mathrm{PG}=\mathrm{C} 01[\mathrm{X}(\mathrm{C} 6 \mathrm{H} 8 \mathrm{~N} 1 \mathrm{O} 1)] \wedge @$

BHandHLYP/aug-cc-pVDZ/BHandHLYP/cc-pVDZ

$\mathrm{HF}=-363.1766876$

\section{References}

1 Frisch, M. J.; Trucks, G. W.; Schlegel, H. B.; Scuseria, G. E.; Robb, M. A.; Cheeseman, J. R.; Zakrzewski, V. G.; Montgomery, Jr., J. A.; Stratmann, R. E.; Burant, J. C.; Dapprich, S.; Millam, J. M.; Daniels, A. D.; Kudin, K. N.; Strain, M. C.; Farkas, O.; Tomasi, J.; Barone, V.; Cossi, M.; Cammi, R.; Mennucci, B.; Pomelli, C.; Adamo, C.; Clifford, S.; Ochterski, J.; Petersson, G. A.; Ayala, P. Y.; Cui, Q.; Morokuma, K.; Malick, D. K.; Rabuck, A. D.; Raghavachari, K.; Foresman, J. B.; Cioslowski, J.; Ortiz, J. V.; Baboul, A. G.; Stefanov, B. B.; Liu, G.; Liashenko, A.; Piskorz, P.; 
Komaromi, I.; Gomperts, R.; Martin, R. L.; Fox, D. J.; Keith, T.; Al-Laham, M. A.; Peng, C. Y.; Nanayakkara, A.; Gonzalez, C.; Challacombe, M.; Gill, P. M. W.; Johnson, B.; Chen, W.; Wong, M. W.; Andres, J. L.; Gonzalez, C.; Head-Gordon, M.; Replogle, E. S.; Pople, J. A. Gaussian 98, Revision A.9; Gaussian, Inc., Pittsburgh PA, 1998.

2 Hehre, W. J.; Radom, L.; Schleyer, P. v. R.; Pople, P. A. Ab Initio Molecular Orbital Theory Wiley: New York, 1986. 